\title{
Simulation in the Front Region of the Earth's Magnetosphere
}

\author{
Gung Yur ${ }^{1, *}$, Ching-Chang Cheng ${ }^{2}$, Chi-Kuang Chao ${ }^{3}$, Jih-Kwin Chao ${ }^{3}$, and Hafiz-Ur Rahman ${ }^{4}$ \\ ${ }^{1}$ Air Asia Co. LTD., Tainan Airport, Taiwan, ROC \\ ${ }^{2}$ Department of Electronic Engineering, National Formosa University, Hu-Wei, Taiwan, ROC \\ ${ }^{3}$ Institute of Space Science, National Central University, Jhongli, Taiwan, ROC \\ ${ }^{4}$ Department of Physics, University of California, Irvine, California, USA
}

Received 3 December 2010, accepted 20 June 2011

\begin{abstract}
A laboratory experiment was conducted to investigate the interaction between a plasma beam and a magnetic dipole, simulating the interaction between the solar wind and magnetized planets. The emphasis in this paper is on the laboratory simulation in the front region of the Earth's magnetosphere and their variation under different solar wind conditions. The boundary in the front region of the magnetosphere is observed in a space simulation laboratory and the magnetospheric structure is produced by a super-Alfvénic and collisionless plasma beam interacting with terrella field. The boundary of the magnetosphere is determined by the factors that include solar wind parameters, such as magnetic fields, ion current density and magnetospheric structure images. It is interesting to compare the results of laboratory simulations with the empirical model by Shue et al. (1997) and the theoretical model by Cheng (1998) as well for the prediction of magnetopause locations under any solar wind condition. The comparisons show that for the northward IMF, magnetopause locations in the laboratory simulation are consistent with the theoretical model. As the magnitude of northward IMF $B_{z}$ becomes higher, the subsolar distance and the flank position in laboratory simulations are consistent with the empirical model as well. For a lower southward IMF $B_{z}$, magnetopause locations in laboratory simulations are consistent with both the empirical and theoretical models. As the magnitude of the southward IMF $B_{z}$ becomes higher, the subsolar distance and the flank position in laboratory simulations seem closer to the theoretical model than the empirical model.
\end{abstract}

Key words: Laboratory simulation, Magnetosphere, Magnetopause

Citation: Yur, G., C. C. Cheng, C. K. Chao, J. K. Chao, and H. U. Rahman, 2012: Simulation in the front region of the Earth's magnetosphere. Terr. Atmos. Ocean. Sci., 23, 85-94, doi: 10.3319/TAO.2011.06.20.02(AA)

\section{INTRODUCTION OF LABORATORY SIMULA- TION}

There are rare experiments and detailed space measurements that have been conducted to date in order to understand the collisionless shock and front region of magnetosphere that are a common feature throughout the universe. Despite the overwhelming amount of data obtained by various missions since then, the basic issues surrounding the physics of such a phenomenon have not yet been fully understood. Also, some of the fundamental properties of the solar wind interaction with the planets are still unresolved. This is mainly because planetary space missions are not common and the data returned from these planetary mis-

\footnotetext{
* Corresponding author

E-mail:yurgung@yahoo.com.tw
}

sions has been limited in many ways. However, an understanding of the solar wind interaction with the Earth is essential to basic planetological problems like the formation of the magnetopause in the front region of magnetosphere. Because of these reasons and high cost of space missions, a fully controllable laboratory simulation experiment may be an attractive approach to study some of the fundamental problems related to the solar wind interaction with planets.

The interaction of flowing magnetized plasma with a stationary dipole magnetic field, simulating the interaction of the solar wind with the Earth, has been studied in a laboratory experiment. The laboratory experiments were performed at the University of California, Riverside (UCRT1) space simulation facility. The facility was upgraded in order to satisfy the scaling requirements (Rahman et al. 
1991). At present, the exact scaling of the relevant parameter ratios from the planetary-magnetosphere to a laboratory experiment is not possible and, perhaps, not even desirable, because of a scaling which is qualitatively similar; nevertheless individual cosmic phenomena can be successfully reproduced and investigated in the laboratory (Podgorny et al. 1970, 1976, 1978). Quantitative differences revealed by continuing research can expand our knowledge of magnetospheres in general and thereby provide additional constraints for the test of mechanisms and models. This unique experiment is not exact simulation of the magnetosphere but does address the formation of each region of the magnetosphere in space physics. The tail region has been studied at the UCR laboratory and observed the X-type and Y-type tail structures for the southward and northward interplanetary magnetic fields (IMF) respectively (Birn et al. 1992) and (Yur et al. 1999). Therefore, we give our attention to the front region of the magnetosphere without comparing the entire global features with the planetary-magnetosphere structures.

Recently, a series of laboratory simulations were conducted to perform magnetosphere-solar wind interactions (Horton and Chiu 2004; Horton et al. 2007; Brady et al. 2009). The solar wind was generated by a laser-target interaction to create supersonic blow-off plasma. Although the magnetopause was observed from their experiments, it is questionable to model the Earth's magnetosphere without the interplanetary magnetic field, which is one of the important characteristics of the solar wind.

Due to the lack of budget support, the UCR-T1 laboratory simulation was terminated in 2000 . The main purpose of this paper is going to conclude and present the unpublished final experimental data from UCR-T1, since the laboratory simulation experiment was promised. Hopefully it will provide following researchers with a reference and guidance in the future.

\section{APPROACH OF LABORATORY SIMULATION}

In this paper, full structures of the magnetopause in a simulated magnetosphere and its location for different polarities and magnitudes of the applied IMF are investigated. The identification of magnetospheric structures in the simulated front region for various solar wind magnetic field orientations was studied. This study involves taking time-integrated images of the entire magnetosphere by using an open shutter camera. Once the locations of the magnetopause are identified for the various conditions of different IMF's magnitude, it will provide guidance for us to take a diagnostic measurement in a limited region. The next step is to obtain detailed measurements and perform an analysis.

For the study of a magnetopause of the magnetosphere, certain key plasma parameters that define the features and characteristics of the magnetopause need be measured first. The UCR space simulation facility was upgraded to a level where most of the global features of the earth's magnetosphere have been successfully simulated (Yur et al. 1995). A schematic of the simulation experiment and coordinate system are shown in Fig. 1. Before interaction and formation of the magnetosphere, Table 1 presents the comparison of the simulation experiment and Earth's magnetosphere for various parameters related to both the laboratory experiment and space plasma in the undisturbed regions. Our previous investigations have established the presence of a magnetopause and regions of trapped hot plasma inside the magnetosphere. This indicates that indeed the Larmor radius and the collision length in these characteristic regions are sufficiently smaller than the typical magnetosphere dimensions, so that magnetohydrodynamics (MHD) should be a reasonable approximation for large-scale structures. The purpose of this part of the investigation is to explore what extent the interaction regions and collisionless processes govern the magnetopause. The primary objective is to measure the thickness and the internal structure of these boundary regions at several locations in combination with the characteristic plasma parameters. If these measurements confirm a collisionless regime for these regions, more detailed studies are planned for future investigation.

There are three essential ingredients for the composition of Earth's magnetosphere in a laboratory simulation.

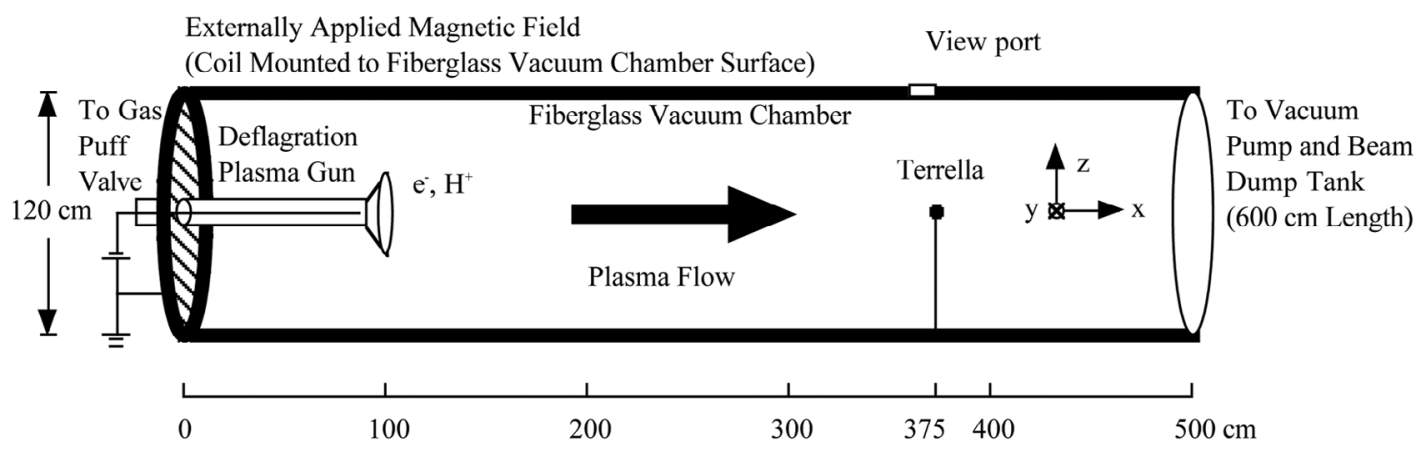

Fig. 1. Schematic of the laboratory experiment setup. 
Table 1. Solar Wind and Earth Parameters (Yur et al. 1999).

\begin{tabular}{|c|c|c|}
\hline Parameter & Space & Laboratory \\
\hline \multicolumn{3}{|l|}{ Solar Wind Parameters } \\
\hline Flow velocity $v_{0}, \mathrm{~cm} \mathrm{~s}^{-1}$ & $4 \times 10^{7}$ & $1 \times 10^{7}$ \\
\hline Plasma density $n_{0}, \mathrm{~cm}^{-3}$ & 5 & $10^{13}-10^{14}$ \\
\hline Electron temperature $T_{e}, \mathrm{eV}$ & $8-10$ & $15-20$ \\
\hline Ion temperature $T_{i}, \mathrm{eV}$ & $4-5$ & $5-10$ \\
\hline Interplanetary magnetic field $B_{I M P}, \mathrm{G}$ & $5 \times 10^{-5}$ & $200-400$ \\
\hline Alfven Mach number $M_{A}$ & 8 & $4-5$ \\
\hline \multicolumn{3}{|l|}{ Earth Parameters } \\
\hline Radius $R_{0}, \mathrm{~cm}$ & $6 \times 10^{8}$ & 3.15 \\
\hline Magnetic field at the equator $B_{e q}, \mathrm{G}$ & 0.3 & $2.2 \times 10^{4}$ \\
\hline Magnetic moment $\mu, \mathrm{G} \mathrm{cm}^{3}$ & $8 \times 10^{25}$ & $6.9 \times 10^{5}$ \\
\hline Magnetospheric size $x_{0}, \mathrm{~cm}$ & $\sim 10^{10}$ & $10-13$ \\
\hline \multicolumn{3}{|l|}{ Dimensionless Parameters } \\
\hline$\beta_{\mathrm{D}}=4 \pi \rho_{0} v_{0}^{2} / B_{0}^{2}=M_{A}^{2}$ & 64 & $\sim 20$ \\
\hline$\beta_{\mathrm{p}}=4 \pi n_{0} T_{0} / B_{0}^{2}$ & 0.6 & $0.3-2.8$ \\
\hline$R_{m}=R_{H}\left(\Omega_{\mathrm{e}} \tau_{\mathrm{e}}\right)$ & $\sim 10^{12}$ & $\sim 10^{3}$ \\
\hline$R_{H}=\beta_{\mathrm{D}}\left(x_{0} / r_{0 i L}\right)$ & $\sim 8 \times 10^{3}$ & $60-200$ \\
\hline$r_{0 i L} / x_{0}$ & $\sim 10^{-3}$ & $0.1-0.3$ \\
\hline$\lambda$ & $\sim 6 \times 10^{-3}$ & $1-3$ \\
\hline
\end{tabular}

The first ingredient is the solar wind, fully ionized hydrogen plasma that streams from the sun. The second is the Earth's magnetic field that has the same form as that of a bar magnet, or dipole magnetic field. The third ingredient, a large-scale interplanetary magnetic field (IMF), plays an important role in the Earth's interaction with the solar wind.

The conditions for magnetizing the model solar wind are studied and verified that the magnetization depends both on the strength of the applied magnetic field and the distance it travels through the magnetic field. After propagating a distance of 3 meters in the interaction vacuum chamber, a minimum strength of about 100 Gauss was found necessary to obtain significant magnetization. The magnetization is characterized by three distinct phases. A brief state of initial diamagnetism is followed by a rapid penetration of the magnetic field into the plasma beam. This is accompanied by a high level of magnetic fluctuations with frequencies close to the lower hybrid frequency, that are identified as whistler modes, not seen on the density and electric field probes. Subsequently the plasma excludes the magnetic flux and the oscillations are damped. Finally the magnetic field penetrates rather slowly into the beam, leading to an $\mathbf{E} \times \mathbf{B}$ propagation. The slow diffusion rate, which is still much faster than the classical diffusion rate, can be explained by Hall conductivity. In this manner we identify the portion of the beam that is fully magnetized and has the parameter regime to scale the real solar wind plasma (Song et al. 1990; Rahman et al. 1991).

A coaxial plasma gun, presently operating in a deflagration mode generates the model solar wind. The electrodes of the gun are directly connected to a $25 \mathrm{kV}$ maximum energy source, and hydrogen is injected to initiate the discharge. The gun is powered by a $500 \mu \mathrm{F}$ capacitor bank which is normally charged to $7-8 \mathrm{kV}$, delivering $200 \mathrm{kA}$ of current. Hydrogen is injected between the gun electrodes when a fast valve is opened electromagnetically by firing a $30 \mu \mathrm{F}$ capacitor bank charged to $5.3 \mathrm{kV}$. A single shot produces a plasma pulse of 70 to $100 \mu$ s duration, flowing through the drift chamber with a velocity of about $4 \times 10^{7} \mathrm{~cm} \mathrm{~s}^{-1}$, measured by time of flight. As the plasma flows downstream from the gun, it expands and fills the tank cross section.

The planetary dipole field is simulated by using a $6.3 \mathrm{~cm}$ diameter spherical wound 10-turn coil embedded in epoxy, powered by a $500 \mu \mathrm{F}$ capacitor bank charged to 6 $10 \mathrm{kV}$, that delivers a dipole field of $20-40 \mathrm{kG}$ for about $200 \mu$ s. The interplanetary magnetic field is simulated by an external coil, wound around the test section and driven by a $500 \mu \mathrm{F}$ capacitor bank charged to $1-6 \mathrm{kV}$. It can produce a vertical ( $z$-axis) magnetic field of a maximum strength of $500 \mathrm{G}$ for a cycle period of $2 \mathrm{~ms}$. The flowing plasma beam gets fully magnetized before it interacts with the dipole field and the flow is super-Alfvénic for most of the pulse duration (Wessel et al. 1990).

Two diagnostics were used to characterize the front region of magnetosphere in this laboratory simulation experiment. One is a two dimensional magnetic probe $\left(B_{x}\right.$ $B_{z}$ ). The most important diagnostic for our magnetospheric simulation experiment is a set of small, orthogonal and hollow dual-axial magnetic search coils. The voltage for each of the two search coils is integrated and digitally recorded. We have developed an automatic probe positioning system that can move the search coils to any desired position inside the simulated magnetosphere in order to investigate its 2-D topology. Another is dual-sided Faraday cups. The voltage-biased Faraday cups (-50 to $-100 \sim \mathrm{V}$ bias) are used to measure ion current density, ion time-of-flight (TOF) velocity (using cups located in sequential ports), plasma beam density (from the velocity), and the beam divergence (by scanning the beam cross section at various downstream locations). We have fabricated very small two-sided Faraday cups that will be used for determining the net flow of plasma in the magnetotail region (Yur et al. 1999).

\section{RESULTS OF LABORATORY SIMULATION}

Figures 2 and 3 display the coordinate system used with $x$ pointing sunward, $z$ pointing northward and $y$ completing 
the right-handed rectangular system, these photographic images of the magnetosphere were taken with an open shutter camera for southward and northward IMF with 200 and $300 \mathrm{G}$ magnitude. The images were digitally analyzed with advanced computational techniques to clarify different structures of the simulated magnetosphere. The light is emitted from background gas that is heated by collisions with the hot dense plasma and thus gives an impression of the regions where denser plasma is present. The images show significant differences in structures for different orientations of the IMF, primarily at the front boundary and in the tail region. The structure of the magnetotail has been investigated in a laboratory simulated magnetosphere (Birn et al. 1992; Yur et al. 1999). The locations of the magnetopause $x=9 \mathrm{~cm}$ and $x=10 \mathrm{~cm}$ for northward $200 \mathrm{G}$ and southward -200 G IMF respectively that corresponds to the location shown in the photographic image of Fig. 2. The locations of the magnetopause $x=11 \mathrm{~cm}$ and $x=9 \mathrm{~cm}$ for northward $300 \mathrm{G}$ and southward $-300 \mathrm{G}$ IMF respectively that corresponds to the location shown in the photographic image of Fig. 3.

The location of the magnetopause is also measured and verified by using a 2-D ( $x$ and $z$ axis components) magnetic probe and a double-sided faraday cup both at $x=6.15 \mathrm{~cm}$ location along the $z$-axis in the front region. Figure 4 shows the total magnetic field $\left(B_{T}\right)$ for southward IMF along the $z$-axis that is basically a north-south line. The jump in the intensity of the magnetic field represents the location of the magnetopause $z=8.5 \mathrm{~cm}$ and $z=12 \mathrm{~cm}$ for 200 and $-200 \mathrm{G}$ IMF respectively that exactly corresponds to the location shown in the photographic image. Figure 5 shows the measurement of earthward ion flux $\left(J_{E W}\right)$ for southward IMF

(a)
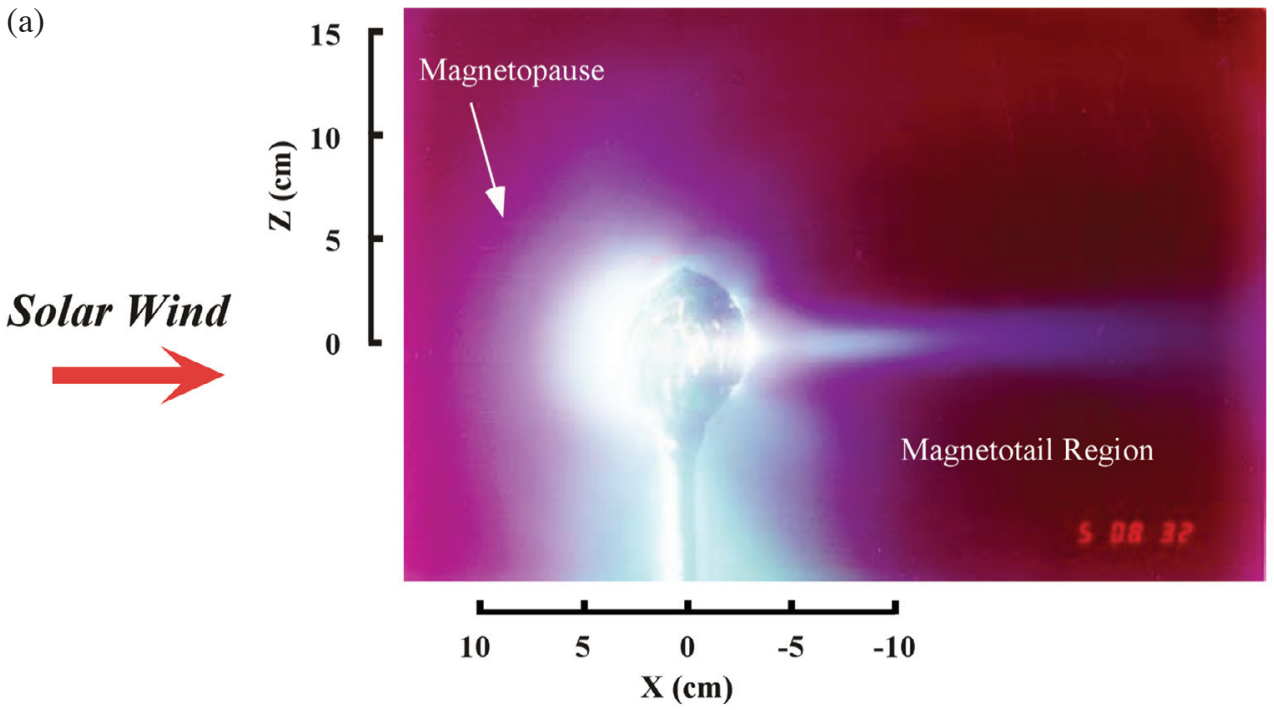

(b)

Solar Wind

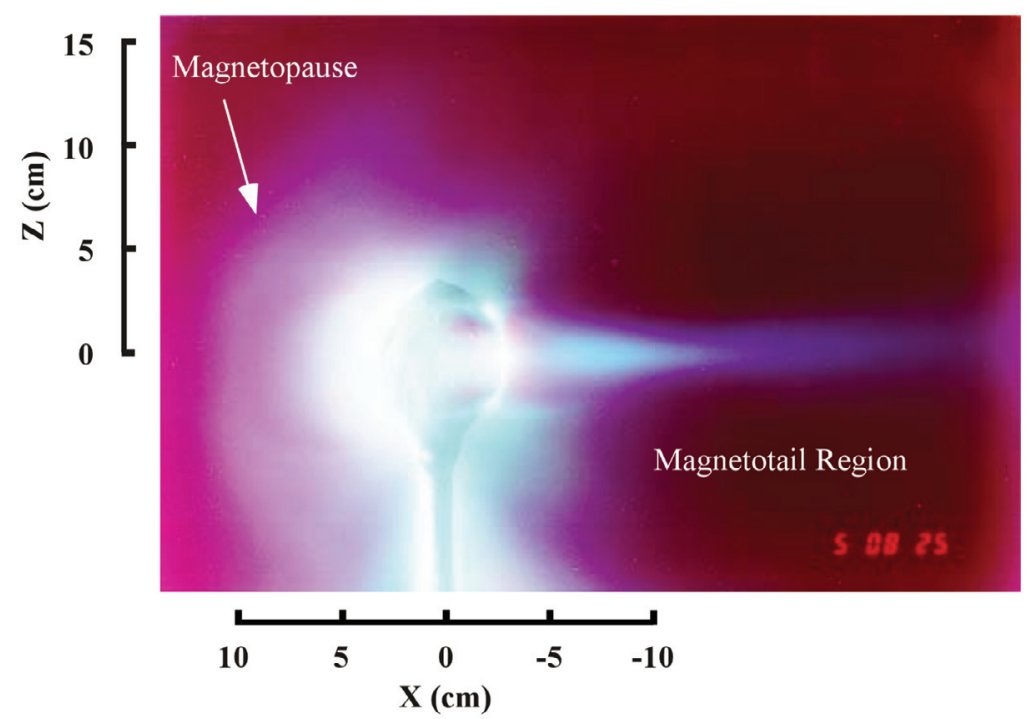

Fig. 2. (a) Photograph of the simulated magnetosphere for northward IMF of 200 G. (b) Photograph of the simulated magnetosphere for southward IMF of $200 \mathrm{G}$. 
(a)

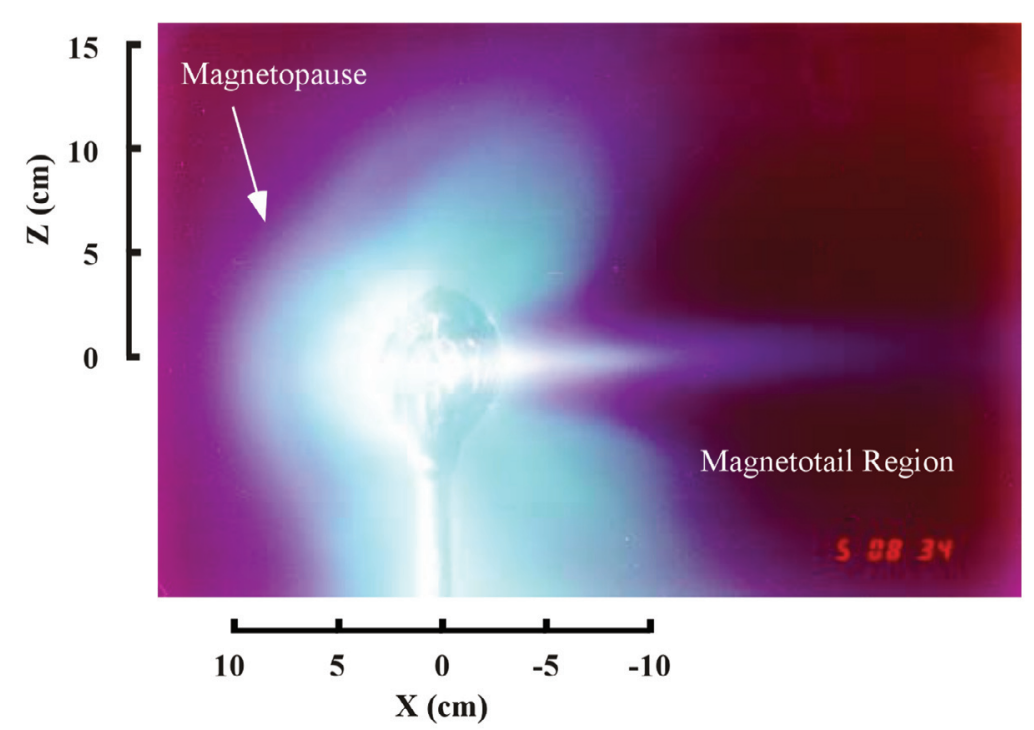

(b)

\section{Solar Wind}
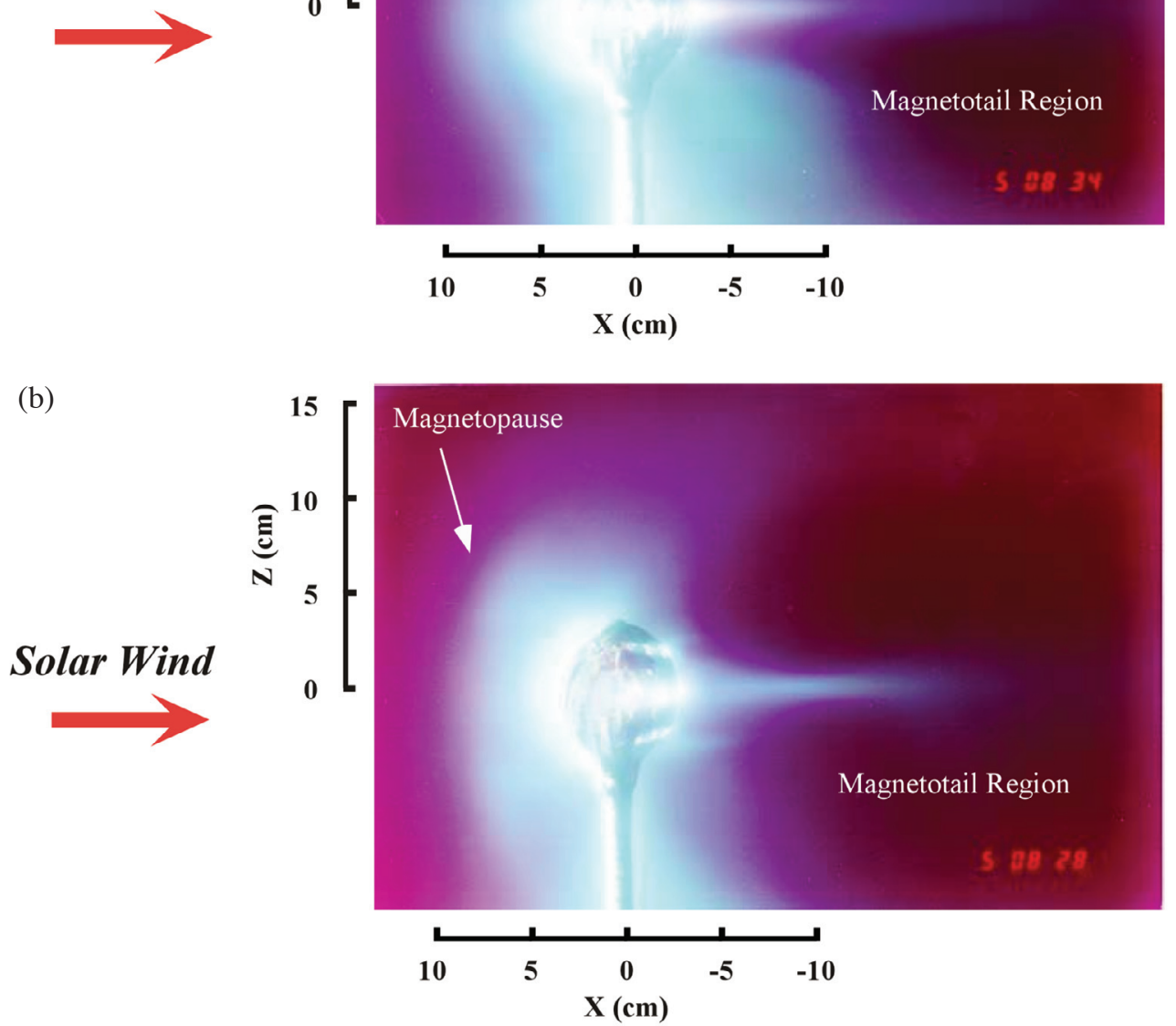

Fig. 3. (a) Photograph of the simulated magnetosphere for northward IMF of 300 G. (b) Photograph of the simulated magnetosphere for southward IMF of $300 \mathrm{G}$.
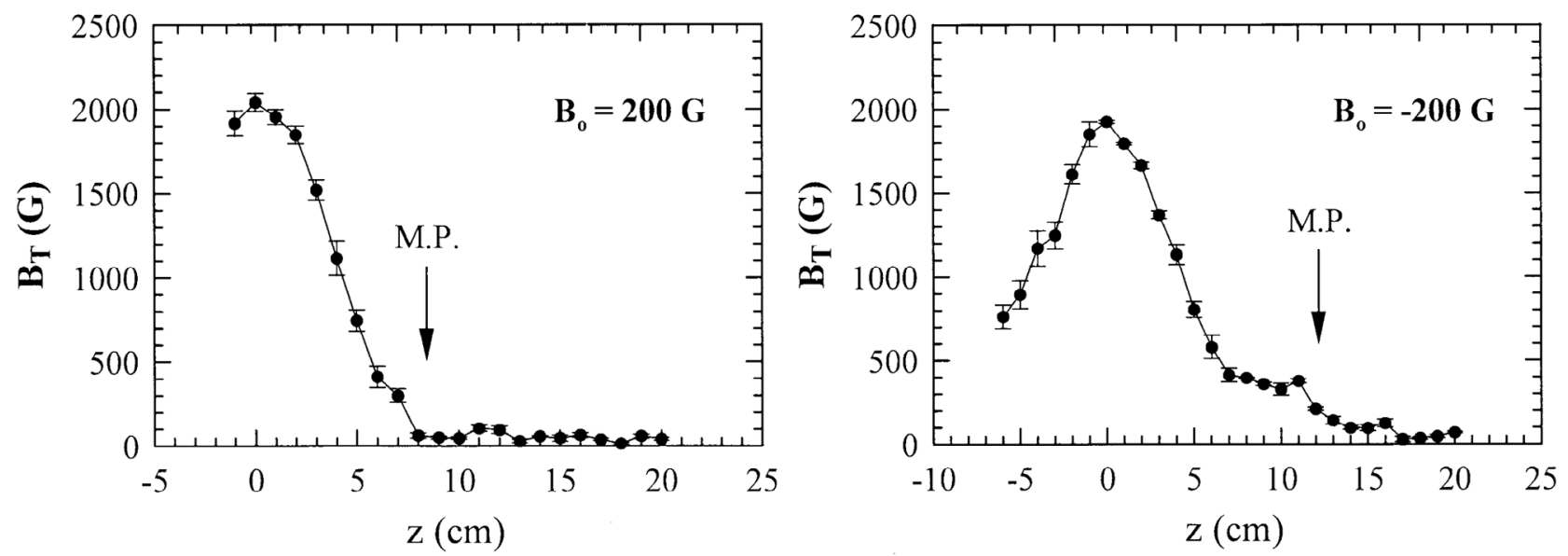

Fig. 4. Total magnetic field $\left(B_{T}\right)$ as function of $z$ measured by a 2-D magnetic probe at $x=6.15 \mathrm{~cm}$ in the front region for the northward and southward IMF $\left(B_{o}=200\right.$ and $\left.-200 \mathrm{G}\right)$. 

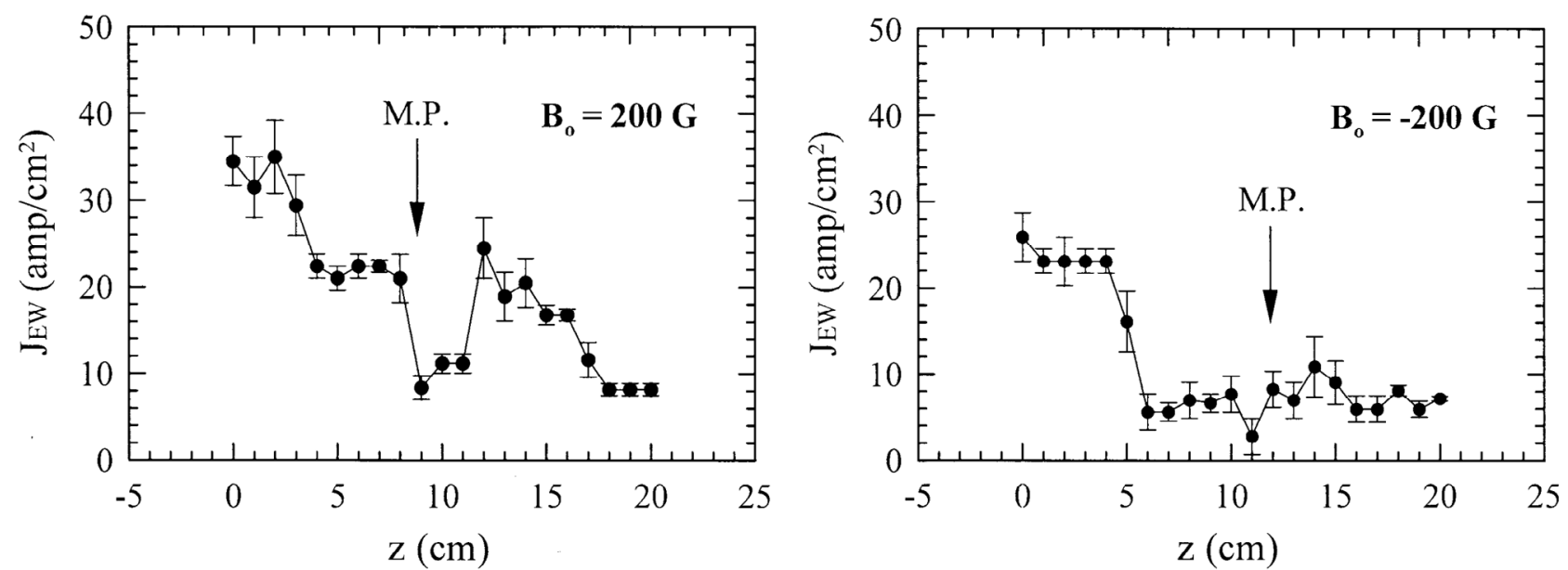

Fig. 5. Earthward ion flux $\left(J_{E W}\right)$ as function of $z$ measured by a 2-D magnetic probe at $x=6.15 \mathrm{~cm}$ in the front region for the northward and southward $\operatorname{IMF}\left(B_{o}=200\right.$ and $\left.-200 \mathrm{G}\right)$.

along the same line showing a distinct jump at the location of the magnetopause. The total magnetic field $\left(B_{T}\right)$ and earthward ion flux $\left(J_{E W}\right)$ for 300 and -300 G IMF along the $z$-axis are shown in Figs. 6 and 7 respectively. When the probes move earthward and pass the magnetopause, there is a trend in Figs. 5 and 7 that the magnetic field increases and the earthward ion flux declines slightly but clearly with decreasing $z$ across the magnetopause boundary for the southward IMF. The distinct jumps in the intensity of $B_{T}$ and $J_{E W}$ also represent the location of the magnetopause $z=9.5 \mathrm{~cm}$ and $z=13 \mathrm{~cm}$ for 300 and $-300 \mathrm{G}$ IMF respectively that exactly corresponds to the location shown in the photographic image.

\section{LABORATORY SIMULATION COMPARED TO THE EMPIRICAL AND THEORETICAL MODELS}

It has long been known that the magnetopause location is mainly controlled by the solar wind dynamic pressure $D_{p}$ and IMF $B_{z}$ component (Fairfield 1995). In order to predict the magnetopause location, Shue et al. (1997) proposed an empirical model that the magnetopause shape can be fitted with a simple functional form. According to Shue et al. (1997), the magnetopause shape can be fitted as

$$
r=r_{o}\left(\frac{2}{1+\cos \theta}\right)^{\alpha}
$$

where $r$ is the radial distance from the Earth's center to the magnetopause, $r_{o}$ is the distance from the Earth's center to the subsolar point and $\alpha$ is the exponential factor related to magnetopause flaring. For the northward IMF, the subsolar distance $r_{o}$ is derived as

$$
r_{o}=\left(11.2+0.013 B_{z}\right)\left(D_{p}\right)^{-1 / 6.6}
$$

where $B_{z}$ in nanoteslas (nT) is the $z$ component of the IMF and $D_{p}$ in nanopascals (nPa) denotes the solar wind dynamic pressure. For the southward IMF,

$r_{o}=\left(11.2+0.14 B_{z}\right)\left(D_{p}\right)^{-1 / 6.6}$

and the flaring factor $\alpha$ is expressed as

$\alpha=\left(0.58-0.01 B_{z}\right)\left(1+0.01 D_{p}\right)$

From Eqs. (2) to (4), $r_{o}$ and $\alpha$ are determined by the IMF $B_{z}$ and $D_{p}$. Thus for given IMF $B_{z}$ and $D_{p}$, magnetopause locations in the laboratory simulation can be used to compare to the Shue et al. (1997) model.

By using the field line topology, Yeh (1997) presented a model magnetosphere that can be described as a closed magnetopause in the dayside and an open magnetotail in the nightside. In addition to the Earth's dipole and a southward IMF, the contribution due to the Chapman-Ferraro current needs to be included. By using the method of images, the effect upon the magnetospheric field by the Chapman-Ferraro current can be regarded as an image dipole placed in front of the dayside magnetopause. Based on the Yeh (1997) model, the shape of the model magnetopause is compared to an empirical model by Shue et al. (1997). It shows that the strength of the image dipole correlates linearly with the solar wind dynamic pressure for given separation between the Earth's dipole and the image dipole (Cheng 1998). Referring to Yeh (1997), the Earth's magnetopause can be regarded as an interface between the magnetospheric cavity and the interplanetary medium. The interface consists of the flux surface that is constructed on the spatial extent of the coplanar field lines leaving/entering the north/south cusp neutral point. According to Cheng (1998), the location of 

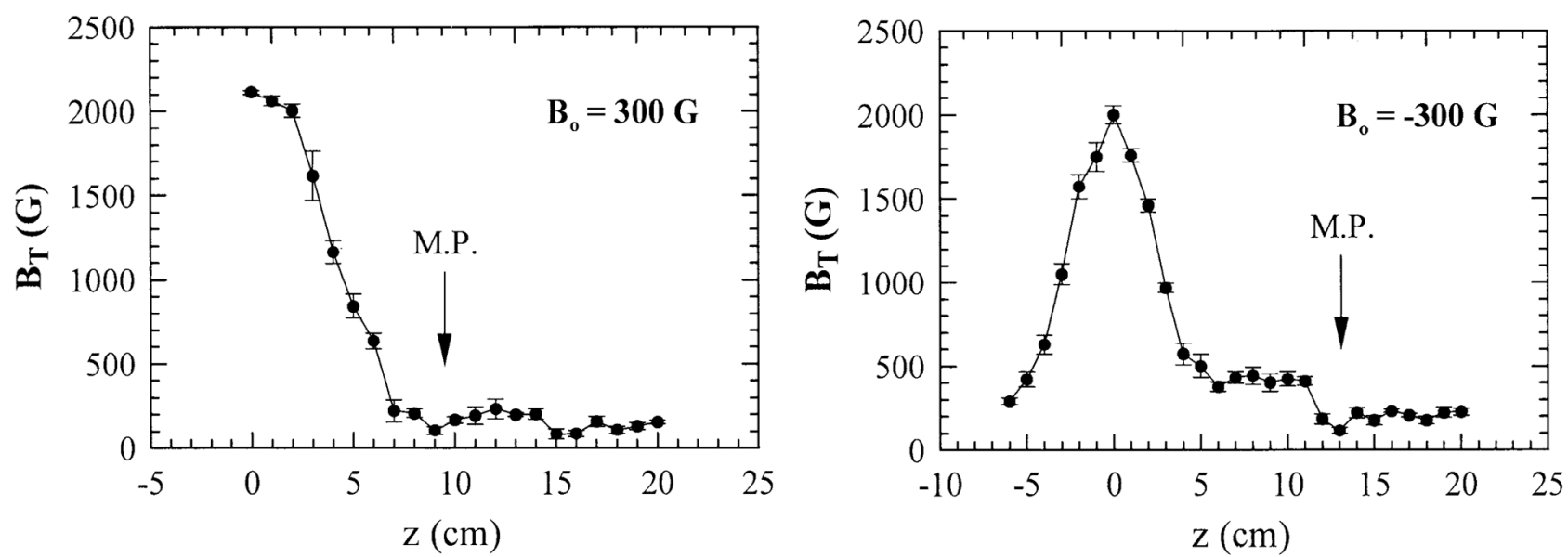

Fig. 6. Total magnetic field $\left(B_{T}\right)$ as function of $z$ measured by a 2-D magnetic probe at $x=6.15 \mathrm{~cm}$ in the front region for the northward and southward IMF $\left(B_{o}=300\right.$ and $\left.-300 \mathrm{G}\right)$.
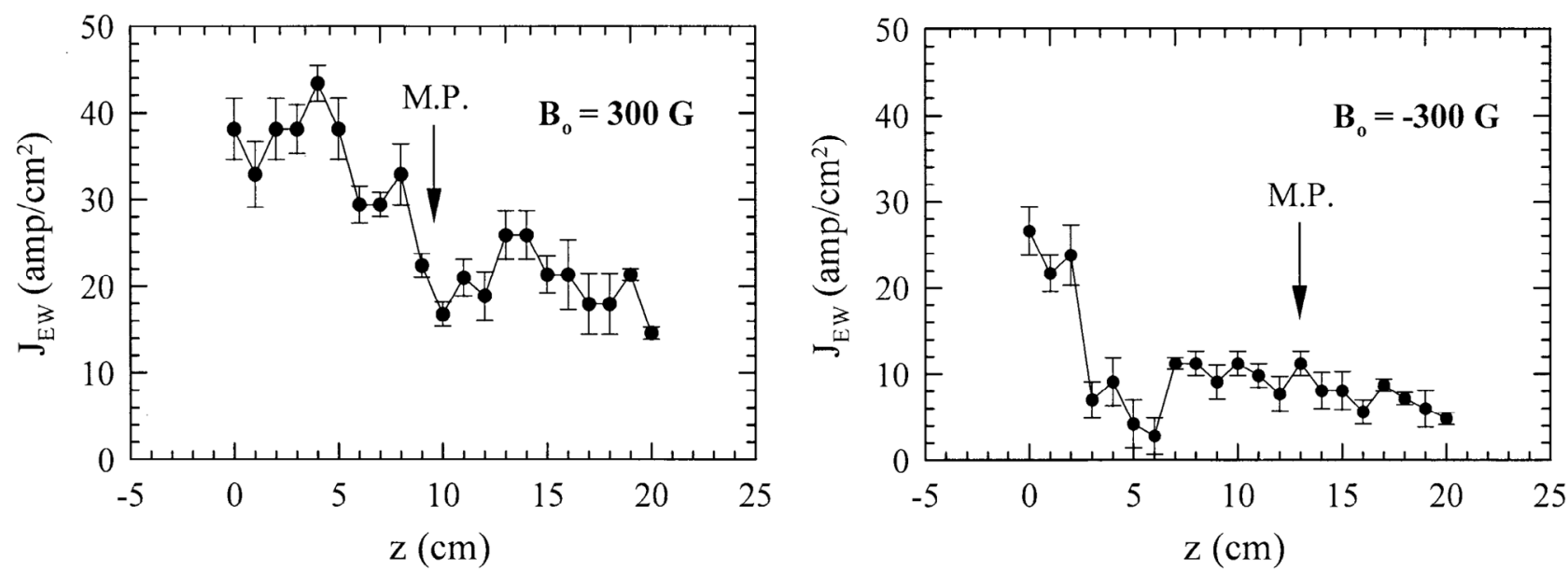

Fig. 7. Earthward ion flux $\left(J_{E W}\right)$ as function of $z$ measured by a 2-D magnetic probe at $x=6.15 \mathrm{~cm}$ in the front region for the northward and southward $\operatorname{IMF}\left(B_{o}=300\right.$ and $\left.-300 \mathrm{G}\right)$.

cusp neutral points is determined by

$\frac{B_{M}}{B_{E}}=\frac{x\left[\left(r_{M}-x\right)^{2}+z^{2}\right]^{5 / 2}}{\left(r_{M}-x\right)\left(x^{2}+z^{2}\right)^{5 / 2}}$

and

$\frac{B_{I}}{B_{E}}=\frac{-r_{M}\left(r_{M} x-x^{2}-2 z^{2}\right) a^{3}}{\left(r_{M}-x\right)\left(x^{2}+z^{2}\right)^{5 / 2}}$

where the origin of Cartesian coordinates is located at the Earth's center, the $x$-axis pointing toward the Sun, the $y$-axis toward the dusk, and the $z$-axis toward geomagnetic north. Moreover, $a$ denotes the Earth's radius, $B_{M}$ denotes the strength of an image dipole, $B_{E}$ is the strength of the magnetic field at the Earth's equator, $r_{M}$ is the distance between the Earth's dipole and an image dipole, and $B_{I}$ denotes the magnitude of IMF $B_{z}$ component and can be converted to SI units by multiplying $B_{I} / B_{E}$ by $B_{E}=3.12 \times 10^{4} \mathrm{nT}$. The integration of field lines is described by a differential equation as follows

$\frac{d x}{B_{x}}=\frac{d y}{B_{y}}=\frac{d z}{B_{z}}=\frac{d s}{B}$

where $(d s)^{2}=(d x)^{2}+(d y)^{2}+(d z)^{2}$ and $B^{2}=B_{x}^{2}+B_{y}^{2}+B_{z}^{2}$. The field lines in the neighborhood of a neutral point are determined by the linearized equation that is derived from Eq. (7) with Taylor's expansion at the neutral point. Thus, the model magnetopause can be constructed with the coplanar field lines emanating/entering from the south/north cusp neutral point. The magnetopause shape consists of field lines emanating from the south cusp neutral point and 
represents the cross section of the model magnetosphere for given values of $r_{M} / a, B_{M} / B_{E}$ and $B_{I}$. Hence, it is interesting in comparing the magnetopause prediction in the laboratory simulation to the Cheng (1998) model.

Figures 2 and 3 show the simulated Earth's magnetosphere with the externally applied IMF $B_{o}$ of which the strengths are 200 and $300 \mathrm{G}$, respectively. In the simulated solar wind, the plasma density $n_{o}$ is $7.6 \times 10^{12} \mathrm{~cm}^{-3}$ and the flow velocity $v_{o}$ is $1.3 \times 10^{7} \mathrm{~cm} \mathrm{~s}^{-1}$ for $B_{o}=200 \mathrm{G}$. As $B_{o}=$ $300 \mathrm{G}$, the simulated $n_{o}$ and $v_{o}$ are $1.98 \times 10^{13} \mathrm{~cm}^{-3}$ and $1.2 \times$ $10^{7} \mathrm{~cm} \mathrm{~s}^{-1}$, respectively. According to Yur et al. (1995), the simulated IMF $B_{p}$ (inside the flowing plasma) in vicinity of the terrella becomes $50 \mathrm{G}$ for $B_{o}=200 \mathrm{G}$. As $B_{o}=300 \mathrm{G}, B_{p}$ becomes $180 \mathrm{G}$. With the scaling law by Yur et al. (1995), the simulated IMF $B_{p}=50 \mathrm{G}$ corresponds to the IMF $B_{z}=$ $5 \mathrm{nT}$ in the vicinity of the Earth's magnetosphere. Moreover, $B_{p}=180 \mathrm{G}$ is for IMF $B_{z}=18 \mathrm{nT}$. For $n_{o}=5 \mathrm{~cm}^{-3}$ and $v_{o}=4 \times 10^{7} \mathrm{~cm} \mathrm{~s}^{-1}$ in the solar wind, the solar wind dynamic pressure $D_{p}$ is about $1 \mathrm{nPa}$ and simulated as $n_{o}=10^{13} \mathrm{~cm}^{-3}$ and $v_{o}=4 \times 10^{7} \mathrm{~cm} \mathrm{~s}^{-1}$ in the laboratory simulation. The latter is deduced with equivalence of the ratio of $D_{p} / P_{B}$ in space to the laboratory simulation where $P_{B}$ denotes magnetic pressure. As $B_{p}=50 \mathrm{G}$ in the laboratory simulation, the simulated $D_{p}$ corresponds to $0.9 \mathrm{nPa}$ in the space for the simulated $n_{o}=7.6 \times 10^{12} \mathrm{~cm}^{-3}$ and $v_{o}=1.3 \times 10^{7} \mathrm{~cm} \mathrm{~s}^{-1}$. For $B_{p}=180 \mathrm{G}$, the simulated $D_{p}$ corresponds to $2.0 \mathrm{nPa}$ in space for the simulated $n_{o}=1.98 \times 10^{13} \mathrm{~cm}^{-3}$ and $v_{o}=1.2 \times$ $10^{7} \mathrm{~cm} \mathrm{~s}^{-1}$.

First, we attempt to obtain the magnetopause shape of the Shue et al. (1997) model with the simulated solar wind parameters IMF $B_{z}$ and $D_{p}$. For the northward and southward IMF, the subsolar distance $r_{o}$ of the Shue et al. (1997) model can be obtained by taking the simulated $D_{p}$ and IMF $B_{z}$ into Eq. (3). Subsequently, $\alpha$ can be obtained by taking the simulated $D_{p}$ and IMF $B_{z}$ into Eq. (4). As a result, the magnetospause shape of the Shue et al. (1997) model can be obtained by taking $r_{o}$ and $\alpha$ into Eq. (1). It is shown in Fig. 8a that the dashed line denotes the magnetospause shape of the Shue et al. (1997) model for the $D_{p}=0.9 \mathrm{nPa}$ and the IMF $B_{z}=5 \mathrm{nT}$. Figure $8 \mathrm{~b}$ is in the same format as Fig. 8a, except for $D_{p}=2.0 \mathrm{nPa}$ and the IMF $B_{z}=18 \mathrm{nT}$. With same $D_{p}$ in Figs. 8a and b, Figs. 8c and d show the magnetopause shape of the Shue et al. (1997) model for the southward IMF $B_{z}=-5 \mathrm{nT}$ and $-18 \mathrm{nT}$, respectively.

Next, we attempt to obtain the magnetopause shape from the Cheng (1998) model for the simulated $D_{p}$ and IMF $B_{z}$. The subsolar distance $r_{o}$ is used to unify the comparison of the laboratory simulation with the empirical and theoretical models. It is shown in Figs. 1a and 2a that for the simulated northward IMF, the various values of $r_{o}$ are $x=9.0$ and $11.0 \mathrm{~cm}$ corresponding to 9.0 and 11.0 in the Earth's radius in space, respectively. On the other hand, Figs. $2 b$ and $3 \mathrm{~b}$ show that for the simulated southward IMF the various values of $r_{o}$ are $x=10.0$ and $9.0 \mathrm{~cm}$ corresponding to 10.0 and 9.0 in the Earth's radius in space, respectively. We note that there is a wider difference of the subsolar distance in the laboratory simulation for northward IMF cases than for southward IMF cases. Hence, we first must find the optimal parameters for the model magnetopause under the southward IMF. In contrast to the empirical magnetopause, Shue et al. (1997) determined by the subsolar distance and the flaring factor controlled by the solar wind dynamic pressure $D_{p}$ and the IMF $B_{z}$, the model magnetopause produced by Cheng (1998) is the flux surface constructed on the spatial extent of coplanar field lines leaving/entering north/south cusp neutral points determined by $r_{M} / a, B_{M} / B_{E}$ and $B_{z}$. The subsolar distance of the model magnetopause cannot directly be obtained as in the empirical model. For the southward IMF $B_{z}=-34 \mathrm{nT}, r_{M} / a=30$ and $B_{M} / B_{E}=14$, the subsolar distance in the Cheng (1998) model is about $r_{o} / a=9.5$ close to that in the laboratory simulation. Note that the cross in Fig. 7 denotes the laboratory simulation. There is a trend in Fig. 3 of Shue et al. (1998) that for the southward IMF the subsolar distance seems more affected by the IMF $B_{z}$ than $D_{p}$. Hence, the magnetopause shapes for the southward IMF $B_{z}=-5$ and $-18 \mathrm{nT}$ are obtained with the same values of $r_{M} / a$ $=30$ and $B_{M} / B_{E}=14$. There is other trend in Fig. 3 presented by Shue et al. (1998) that for the northward IMF the subsolar distance is more controlled by $D_{p}$ rather than the IMF $B_{z}$. Thus with $r_{M} / a=30$, the magnetopause shapes for the northward IMF $B_{z}=5$ and $18 \mathrm{nT}$ are obtained by adopting $B_{M} / B_{E}$ $=18$ and 30 which increase in linear proportion as $D_{p}$ enhances, respectively. As a result, Fig. 8 shows that the solid line denotes the magnetopause shape of the Cheng (1998) model for the northward and southward IMF, respectively.

It is shown in Fig. 8a that for the northward IMF $B_{z}=$ $5 \mathrm{nT}$, magnetopause locations in the laboratory simulation are consistent with those in the Cheng (1998) model. From Fig. 8a, the magnetopause shape of the Shue et al. (1997) model becomes more sunward than the laboratory simulation for the lower northward IMF $B_{z}$. For higher northward IMF $B_{z}$, however, Fig. $8 \mathrm{~b}$ shows that the subsolar distance in the laboratory simulation seems more sunward than those found in the Shue et al. (1997) and Cheng (1998) models. Figure $8 \mathrm{c}$ shows that for the southward IMF $B_{z}=-5 \mathrm{nT}$, magnetopause locations in the laboratory simulation are consistent with both the empirical and theoretical models. But as the southward IMF $B_{z}=-18 \mathrm{nT}$, it is shown in Fig. 8d that the subsolar distance and the flank in the laboratory simulation become closer to the Cheng (1998) model than the Shue et al. (1997) model.

\section{CONCLUSIONS}

Our laboratory simulation results show that in the southward IMF case, the flank position of the Earth's magnetopause is extended and the front position contracted due to magnetic field reconnection. In the northward IMF case, 
(a)

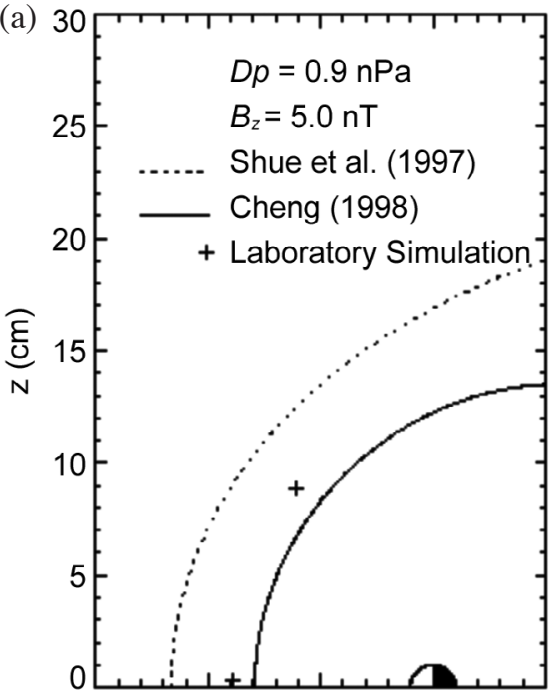

(c)



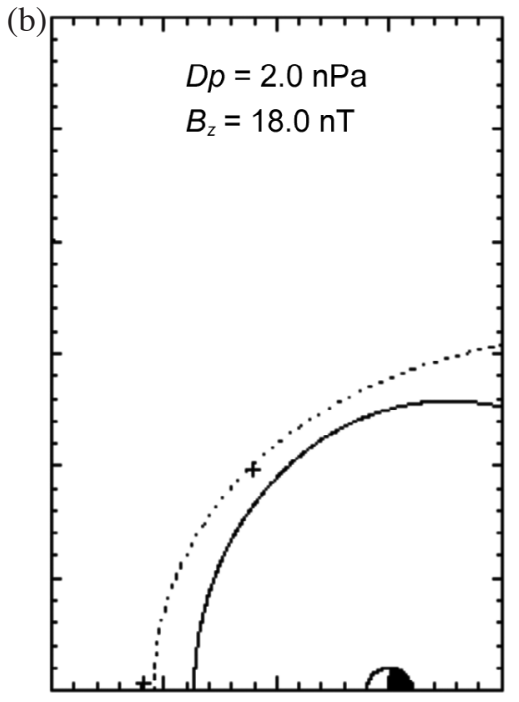

$(\mathrm{d})$

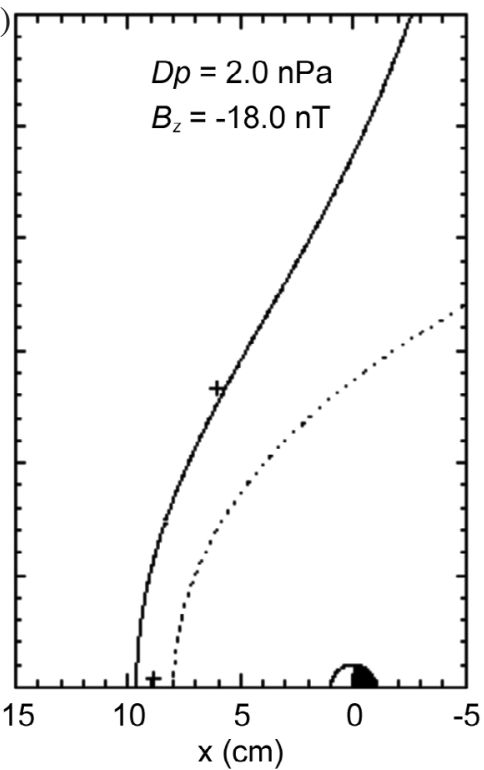

Fig. 8. (a) Magnetopause locations in the laboratory simulation compared to the empirical and theoretical models for the solar wind dynamic pressure $D_{p}=0.9 \mathrm{nPa}$ and IMF $B_{z}=5.0 \mathrm{nT}$. The dotted line denotes the Shue et al. (1997) model. The solid line denotes the Cheng (1998) model. The cross denotes the laboratory simulation. (b) In the same format as (a), except for $D_{p}=2.0 \mathrm{nPa}$ and IMF $B_{z}=18.0 \mathrm{nT}$. (c) In the same format as (a), except for $D_{p}=0.9 \mathrm{nPa}$ and IMF $B_{z}=-5.0 \mathrm{nT}$. (d) In the same format as (a), except for $D_{p}=2.0 \mathrm{nPa}$ and IMF $B_{z}=-18.0 \mathrm{nT}$.

the flank position moves slightly outward along $z$ and the front position of the Earth's magnetopause expanded by solar wind dynamic pressure. They are all caused by the variation of solar wind parameters.

Unlike those deployed in space, more probes in the laboratory simulation can lead to an inevitable distortion of the simulated magnetosphere and thus their measurements cannot be reliable. Despite the lack of abundant data available, the present study can compare qualitatively the laboratory simulation to the theoretical and empirical models to examine which kind of solar wind conditions result in the simulated magnetosphere. Hence, it is plausible that the presented data can support a qualitative interpretation and conclusion.
In summary, the comparisons show that for northward IMF, magnetopause locations in the laboratory simulation are consistent with the theoretical model. As the magnitude of northward IMF $B_{z}$ becomes higher, the subsolar distance and the flank in the laboratory simulation are consistent with the empirical model as well. For a lower southward IMF $B_{z}$, magnetopause locations in the laboratory simulation are consistent with both the empirical and theoretical models. As the magnitude of southward IMF $B_{z}$ becomes higher, the subsolar distance and the flank in the laboratory simulation seem closer to the theoretical model than the empirical model. A possible reason of this result is that both the laboratory simulation and theoretical modeling are based on interaction of the solar wind with a simulated magnetic 
dipole. Therefore, it is expected that the empirical model would produce a different result.

Acknowledgements This work in UCR was supported by LANL/IGPP grant. Los Alamos portions of this work were also supported through the office of the US Department of Energy for Basic Energy Sciences. The work of C. C. Cheng and J. K. Chao was supported by the National Science Council of ROC in Taiwan under grant NSC89-2111M-150-001.

\section{REFERENCES}

Birn, J., G. Yur, H. U. Rahman, and S. Minami, 1992: On the termination of the closed field line region of the magnetotail. J. Geophys. Res., 97, 14833-14840, doi: 10.1029/92JA01145. [Link]

Brady, P., T. Ditmire, W. Horton, M. L. Mays, and Y. Zakharov, 2009: Laboratory experiments simulating solar wind driven magnetospheres. Phys. Plasmas, 16, 043112, doi: 10.1063/1.3085786. [Link]

Cheng, C. C., 1998: The solar wind control of the magnetopause shape: A comparison of a model magnetopause and empirical models. Terr. Atmos. Ocean. Sci., 9, 239-254.

Fairfield, D. H., 1995: Observations of the shape and location of the magnetopause: A review. In: Song, P., B. U. O. Sonnerup, and M. F. Thomsen (Eds.), Physics of the Magnetopause, American Geophysical Union, 53-60.

Horton, W. and C. Chiu, 2004: Laser Z-pinch dipole-target experiments to simulate space physics acceleration processes. Phys. Plasmas, 11, 1645, doi: 10.1063/1.166 6509. [Link]

Horton, W., C. Chiu, T. Ditmire, P. Valanju, R. Presura, V. V. Ivanov, Y. Sentoku, V. I. Sotnikov, A. Esaulov, N. L. Galloudec, T. E. Cowan, and I. Doxas, 2007: Laboratory simulation of magnetospheric plasma shocks. Adv. Space Res., 39, 358-369, doi: 10.1016/j.asr.2005. 01.087. [Link]

Podgorny, I. M., 1976: Laboratory experiments (plasma intrusion into the magnetic field). In: Williams, D. J. (Ed.), Physics of Solar Planetary Environments, p. 241, American Geophysical Union.

Podgorny, I. M. and R. Z. Sagdeev, 1970: Physics of intro- ducing plasma and laboratory experiments. Sov. Phys. Uspekhi, 98, 445.

Podgorny, I. M., E. M. Dubinin, and Y. N. Potanin, 1978: The magnetic field on the magnetospheric boundary from laboratory simulation data. Geophys. Res. Lett., 5, 207-210, doi: 10.1029/GL005i003p00207. [Link]

Rahman,H.U., G. Yur, R. S. White, J. Birn, and F.J. Wessel, 1991: On the influence of the magnetization of a model solar wind on a laboratory magnetosphere. J. Geophys. Res., 96, 7823-7829, doi: 10.1029/90JA01993. [Link]

Rahman, H. U., G. Yur, R. S. White, F. J. Wessel, J. J. Song, and N. Rostoker, 1991: Propagation of plasma beams across the magnetic field. Proc. SPIE, 1407, doi: 10.11 17/12.43532. [Link]

Shue, J. H., J. K. Chao, H. C. Fu, C. T. Russell, P. Song, K. K. Khurana, and H. J. Singer, 1997: A new functional form to study the solar wind control of the magnetopause size and shape. J. Geophys. Res., 102, 94979511, doi: 10.1029/97JA00196. [Link]

Shue, J. H., P. Song, C. T. Russell, J. T. Steinberg, J. K. Chao, G. Zastenker, O. L. Vaisberg, S. Kokubun, H. J. Singer, T. R. Detman, and H. Kawano, 1998: Magnetopause location under extreme solar wind conditions. J. Geophys. Res., 103, 17691-17700, doi: 10.1029/98J A01103. [Link]

Song, J. J., F. J. Wessel, G. Yur, H. U. Rahman, N. Rostoker, and R. S. White, 1990: Fast magnetization of a high-to-low beta plasma beam. Phys. Fluids, 2, 24822486, doi: 10.1063/1.859512. [Link]

Wessel, F. J., J. H. Song, N. Rostoker, G. Yur, and H. U. Rahman, 1990: Fast magnetization of a low to high beta plasma beam, Proceedings SPIE-The International Society for Optical Engineering, 1226, 457, doi: 10.1109/PLASMA.1990.110713. [Link]

Yeh, T., 1997: Magnetospheric structure with a southward interplanetary magnetic field. J. Geophys. Res., 102, 51-56, doi: 10.1029/96JA02043. [Link]

Yur, G., H. U. Rahman, J. Birn, F. J. Wessel, and S. Minami, 1995: Laboratory facility for magnetospheric simulation. J.Geophys. Res., 100, 23727-23736, doi: 10.1029/ 95JA01162. [Link]

Yur, G., T. F. Chang, H. U. Rahman, J. Birn, and C. K. Chao, 1999: Magnetotail structures in a laboratory magnetosphere. J. Geophys. Res., 104, 14517-14528, doi: 10.10 29/98JA02193. [Link] 